\title{
Investigating the efficacy of bisphosphonates treatment against multiple myeloma induced bone disease using a computational model
}

\author{
Bing Ji ${ }^{\mathrm{a},}{ }^{*}$, Qing Yang ${ }^{\mathrm{b}}$, Paul G Genever ${ }^{\mathrm{c}}$ and Michael J Fagan ${ }^{\mathrm{d}}$ \\ ${ }^{a}$ School of Control Science and Engineering, Shandong University, 17923 Jingshi Road, Jinan, \\ 250061, People's Republic of China \\ ${ }^{\mathrm{b}}$ Shandong Provincial Hospital Affiliated to Shandong University, 324 Jingwuweiqi Road, Jinan, \\ 250021, People's Republic of China \\ ${ }^{\mathrm{c}}$ Department of Biology, University of York, Heslington, York, YO10 5DD, UK \\ ${ }^{\mathrm{d}}$ School of Engineering, University of Hull, Cottingham Road, Hull, HU6 7RX, UK
}

\begin{abstract}
Multiple myeloma (MM)-induced bone disease is mortal for most MM patients. Bisphosphonates are first-line treatment for MM-induced bone disease, since it can inhibit osteoclast activity and the resultant bone resorption by suppressing the differentiation of osteoclast precursors into mature osteoclasts, promoting osteoclast apoptosis and disrupting osteoclast function. However, it is still unclear whether bisphosphonates have an anti-tumour effect. In our previous work, a computational model was built to simulate the pathology of MM-induced bone disease. This paper extends this proposed computational model to investigate the efficacy of bisphosphonates treatment and then clear the controversy of this therapy. The extended model is validated through the good agreement between simulation results and experimental data. The simulation results suggest that bisphosphonates indeed have an anti-tumour effect.
\end{abstract}

Keywords: Multiple myeloma, MM-induced bone disease, bisphosphonates, anti-tumour, computational model

\section{Introduction}

Multiple myeloma (MM), a haematological malignancy developed in the bone marrow, is the most frequent cancer involving bone and the second most common cancer involving blood cells [1]. Bone disease, as a major complication of MM, is a fatal danger for MM patients. Up to $60 \%$ of MM patients suffer a fracture during the disease, and MM induced bone destruction rarely heals [2-5]. Bisphosphonates are first-line treatment for MM-induced bone disease [6]. Bisphosphonates are able to target high turnover skeletal sites and then bind to the mineralized bone matrix within these sites. After they are internalized by osteoclasts, bisphosphonates can inhibit osteoclast activity and the resultant bone resorption by suppressing the differentiation of osteoclast precursors into mature osteoclasts, promoting osteoclast apoptosis and disrupting osteoclast function. However, the further investigation is required

${ }^{*}$ Corresponding author: Bing Ji, School of Control Science and Engineering, Shandong University, 17923 Jingshi Road, Jinan, 250061, People's Republic of China. Tel.: 86-0531-88396816-806; Fax:+86-0531-88396813; E-mail: b.ji@sdu.edu.cn.

0959-2989/14/\$27.50 @ 2014 - IOS Press and the authors. 
to investigate the anti-tumour effects of bisphosphonates. Several preclinical and clinical data suggest that bisphosphonates may also have a direct anti-tumour effect or an indirect anti-tumour effect $[7,8]$. However, several studies provide contradictory results [9].

Computational modelling has demonstrated great potential in biological study [10-15]. In this paper, a previously developed computational model of MM-induced bone disease by Ji et al. [14] is extended to simulate bisphosphonates treatment against MM-induced bone disease. The simulation results can be used to investigate the efficacy of bisphosphonates and clear the controversy of the treatment.

\section{Model development}

The computational model proposed by Ji et al. [14] contains five variables (osteoblast precursors $\left(O B_{p}\right)$, active osteoblasts $\left(O B_{a}\right)$, active osteoclasts $\left(O C_{a}\right)$, active MM cells $(M M)$ concentrations and bone volume $(B V))$ and is made up of following equations:

$$
\begin{aligned}
& \frac{d O B_{p}}{d t}=D_{O B_{u}} \cdot \pi_{a c t, O B_{u}}^{T G F \beta} \cdot O B_{u}-D_{O B_{p}} \cdot \pi_{r e p, O B_{p}}^{T G F \beta} \cdot \pi_{r e p, O B_{p}}^{V C A M 1} \cdot O B_{p} \\
& \frac{d O B_{a}}{d t}=D_{O B_{p}} \cdot \pi_{r e p, O B_{p}}^{T G F \beta} \cdot \pi_{r e p, O B_{p}}^{V C A M 1} \cdot O B_{p}-A_{O B_{a}} \cdot \pi_{a c t, O B_{a}}^{V C A M 1} \cdot O B_{a} \\
& \frac{d O C_{a}}{d t}=D_{O C_{p}} \cdot \pi_{a c t, O C_{p}}^{R A N L L} \cdot O C_{p}-\pi_{a c t, O C_{a}}^{T G F \beta} \cdot A_{O C_{a}} \cdot O C_{a} \\
& \frac{d M M}{d t}=D_{M M} \cdot \pi_{a c t, M M}^{I L 6} \cdot \pi_{a c t, M M}^{V C A M 1} \cdot M M \cdot\left(1-\frac{M M}{M M_{m a x}}\right)-A_{M M} \cdot \pi_{r e p, M M}^{S L R P S} \cdot M M \\
& \frac{d B V}{d t}=-K_{r e s} \cdot O C_{a}+K_{f o r m} \cdot O B_{a}
\end{aligned}
$$

which describe the temporal variations in $O B_{p}, O B_{a}, O C_{a}, M M$ concentrations and $B V$ respectively. The model uses 'Hill functions' to describe the cellular interaction via the single ligand to receptor binding through $\pi$ functions [16]. The definitions of model parameters including $O B_{u}$, $O C_{p}, M M_{\text {max }}, K_{\text {res }}, K_{\text {form }}, T G F \beta, R A N K L, V C A M 1, I L 6$ and SLRPs are included in [14].

As shown in Eqs. (6) and (7), 'Hill functions' can both describe the stimulating and inhibiting functions of the ligand-receptor binding in the forms of $\pi_{\text {act,receptor }}^{\text {ligand }} \pi_{r e p, \text { receptor }}^{\text {ligand }}$, respectively. ligand in $\pi$ functions represents the concentration of ligand in the ligand-receptor binding. $q, p_{1}, p_{2}$ and $c$ are parameters related with $\pi$ functions, and their definitions can be found in [14].

$$
\begin{aligned}
& \pi_{\text {act }, \text { receptor }}^{\text {ligand }}=\frac{q \cdot(\text { ligand })^{c}}{p_{1}+(\text { ligand })^{c}} \\
& \pi_{\text {rep }, \text { receptor }}^{\text {ligand }}=\frac{q}{1+\left(\frac{\text { ligand }}{p_{2}}\right)^{c}}
\end{aligned}
$$

In the simulation, the possible direct anti-tumour effects of bisphosphonates are not included in the model, since the further investigation is required to confirm this point. The model only considers the role of bisphosphonates inhibiting bone resorption by suppressing the differentiation of mature osteoc 


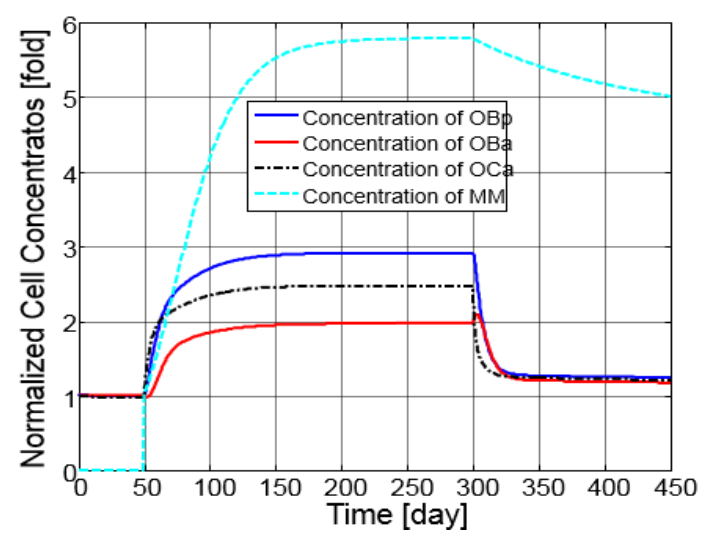

Fig. 1. The variation of normalized cell concentrations with respect to their initial value during different periods: the normal (healthy) period from day 1 to day 50 , the invasion of MM cells from day 51 to day 300 and the intervention of the bisphosphonates therapy from day 301 .

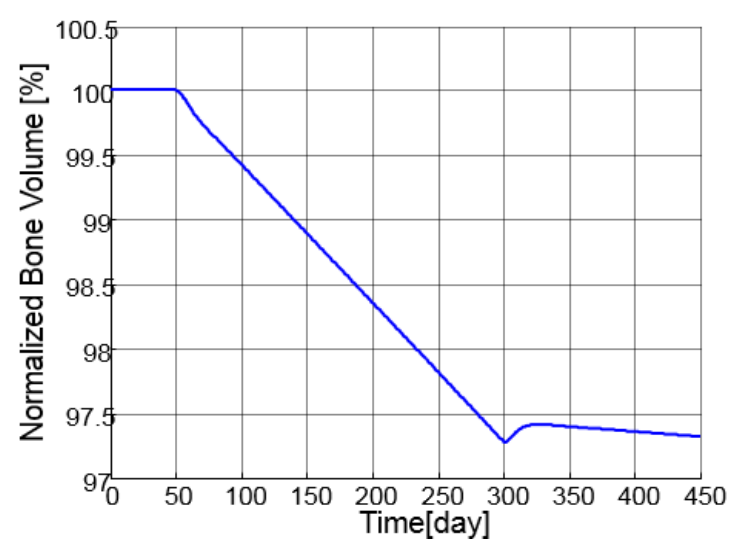

Fig. 2. The variation of normalized bone volume with respect to its initial value during different periods: the normal period from day 1 to day 50, the invasion of MM cells from day 51 to day 300 and the intervention of the bisphosphonates therapy from day 301 .

lasts as well as promoting the apoptosis of osteoclasts. Thus a parameter ' $\mathrm{F}$.Bi' which represents the degree that the bisphosphonates inhibit bone resorption, is introduced in Eq. (3) to represent the underlying mechanism of bisphosphonates treatment. The new equation is presented as follows:

$$
\frac{d O C_{a}}{d t}=D_{O C_{p}} \cdot \pi_{a c t, O C_{p}}^{R A N K L} \cdot O C_{p} \cdot \mathrm{F} . \mathrm{Bi}-\pi_{a c t, O C_{a}}^{T G F \beta} \cdot A_{O C_{a}} \cdot O C_{a} \cdot(1+(1-\mathrm{F} . \mathrm{Bi}))
$$

for example, when ' $\mathrm{F}$.Bi' is set as 0.7 , it means that the differentiation rate of active osteoclasts decreases to $70 \%(0.7)$, while the apoptosis of osteoclasts increases by $30 \%(0.3=1-0.7)$. The previous computational model is extended to simulate the efficacy of bisphosphonates treatment through updating Eq. (3) by Eq. (8).

\section{Simulation results}

In this work, a genetic algorithm is implemented to estimate unknown model parameters. The Runge-Kutta (4th and 5th order) integration method, whose corresponding Matlab solver is ode 45, is selected to solve the model equations. The parameters estimation and model equations solution are carried out in Matlab software (version: 7.6.0) [14]. The model solutions represent variations of in $O B_{p}, O B_{a}, O C_{a}, M M$ concentrations and $B V$ with time respectively.

Figures 1-3 demonstrate how a bisphosphonates therapy would influence cell concentrations and bone volume $(\mathrm{F} . \mathrm{Bi}=0.7)$. From day 1 to day 50 , the bone microenvironment stays in the stable state where cell concentrations of $O B_{p}, O B_{a}$ and $O C_{a}$, and bone volume nearly keep constant. MM cells appear from day 51, disturb the stable state of the bone microenvironment and consequently result in the variation of cell concentrations and bone volume. Figure 1 indicates that the bisphosphonates therapy reduces MM concentrations by $16 \%$ (for the period considered) and helps bone cell concentrations 


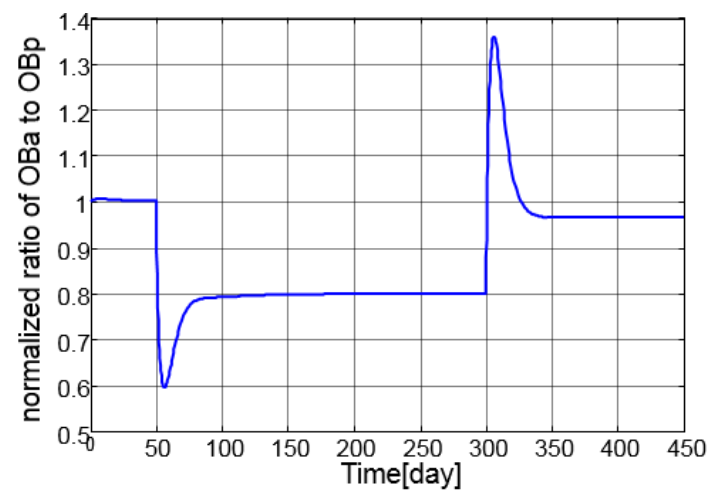

Fig. 3. The variation of normalized ratio of $\mathrm{OBa}: \mathrm{OCa}$ with respect to its initial value during different periods: the normal period from day 1 to day 50, the invasion of MM cells from day 51 to day 300 and the intervention of the bisphosphonates therapy from day 301 .

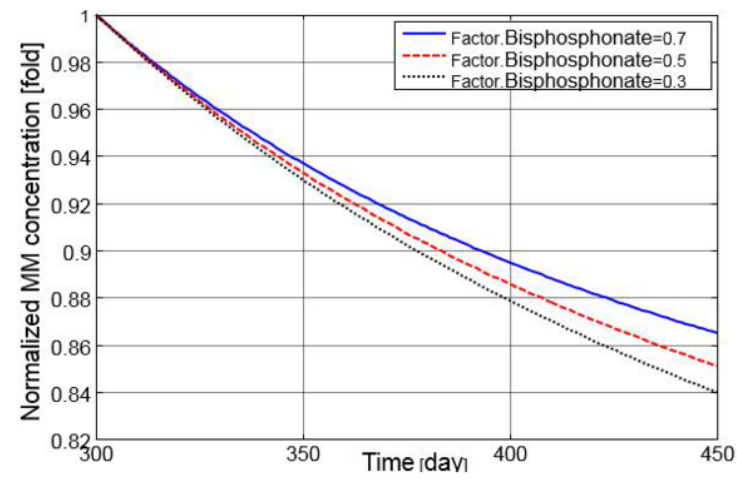

Fig. 4. The variation of normalized MM concentration with respect to the value at day 300 after use of the bisphosphonates therapy with different values of ' $\mathrm{F}$.Bi'.

return to their normal values (i.e. values before the invasion of tumour cells). It is should be noted that the anti-tumour effects of bisphosphonates are not considered. Therefore the decreased tumour burden is due to the inhibited osteoclast activity by bisphosphonates, which agrees with the experimental conclusion that the decrease in osteoclast activity can inhibit the proliferation of MM cells [16,17]. As illustrated in Figure 3, the OBa:OCa ratio increases by 23\% after the introduction of the bisphosphonates therapy, which thus results in a significant slowdown of the bone destruction (shown in Figure 2). Again, this is confirmed by published data that shows bisphosphonates are beneficial to the suppression of MM-induced bone destruction $[18,19]$.

Figures 4-6 show the variations of $\mathrm{MM}$ concentration, bone volume and $\mathrm{OB}_{\mathrm{a}}: \mathrm{OC}_{\mathrm{a}}$ ratio caused by bisphosphonates with different values of ' $\mathrm{F}$.Bi' $(0.7,0.5$ and 0.3$)$ for the same treatment strategy. MM concentration decreases to $86.8 \%, 85.2 \%$ and $84 \%$ of its value at day 300 (for the period considered), when 'Factor.Bisphosphonate' is set as $0.7,0.5$ and 0.3 (shown in Figure 4). As illustrated in Figure 5, when ' $\mathrm{F}$.Bi' is set as 0.7 , the bone destruction continues although its rate is decreased dramatically, however when ' $\mathrm{F}$. Bi' is set to 0.5 or 0.3 , the bone destruction stops and bone volume begins to increase. Thus, the simulation results suggest that a smaller ' $\mathrm{F}$.Bi' produces more significant inhibition of MM concentration and bone destruction. The decreased or ceased bone destruction shown in Figure 5 is due to the increasing $\mathrm{OB}_{\mathrm{a}}: \mathrm{OC}_{\mathrm{a}}$ ratio caused by the bisphosphonate treatment demonstrated in Figure 6.

In Figures 1-6, the bisphosphonates treatment ends on day 450. The reason why day 450 is chosen as terminal time is that as demonstrated in Figures 1 and 3, the concentrations of $O B_{p}, O B_{a}$ and $O C_{a}$, and OBa:OCa ratio nearly keep stable around day 450 , and after day 450 the trends of curves regarding cell concentrations, bone volume (as shown in Figure 7) and OBa:OCa ratio keep the same pattern (the simulation of $\mathrm{OBa}: \mathrm{OCa}$ ratio after day 450 is not given, because it can be calculated based on the data from the first figure in Figure 7). 


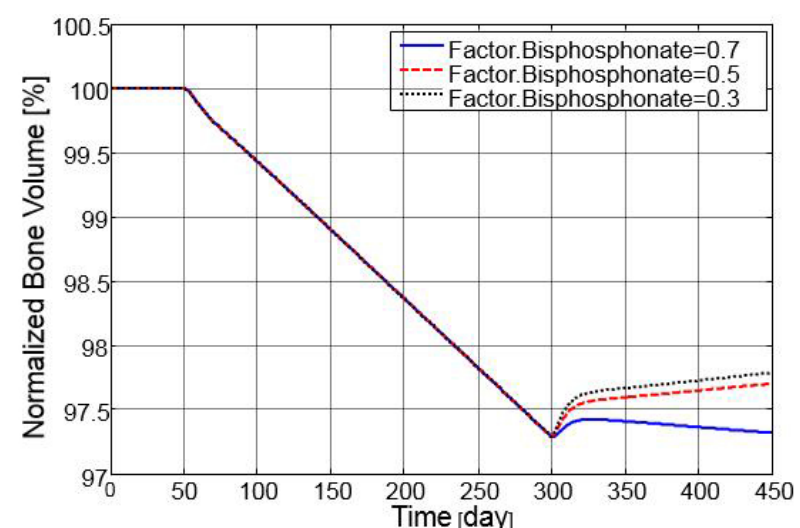

Fig. 5. The variation of normalized bone volume with respect to its initial value after use of the bisphosphonate therapy with different values of ' $\mathrm{F}$.Bi'.

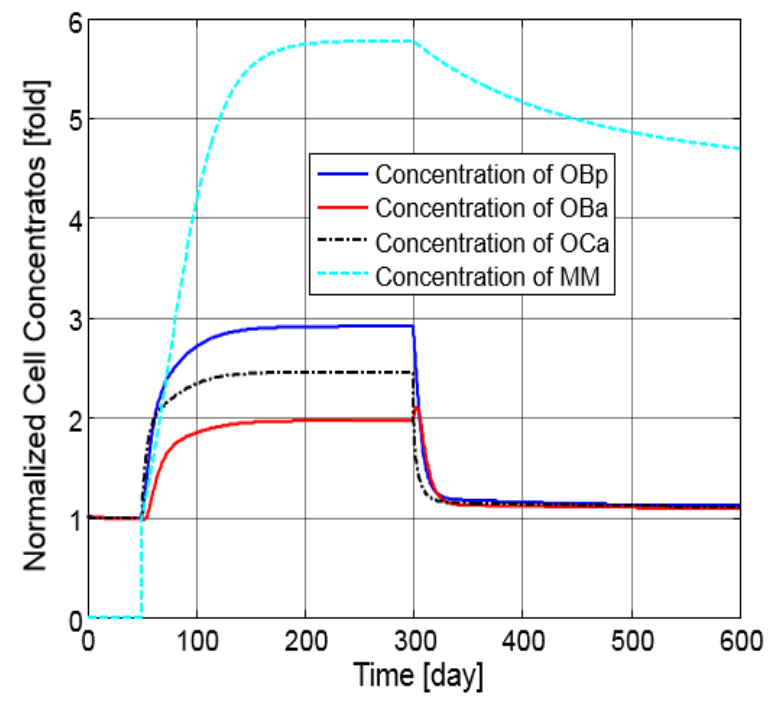

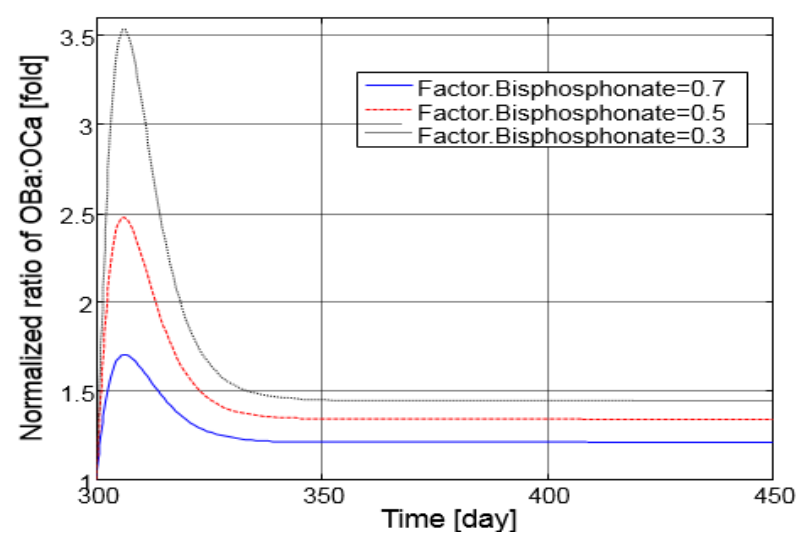

Fig. 6. The variation of normalized ratio of OBa:OCa with respect to the value at day 300 after use of the bisphosphonate therapy with different values of ' $\mathrm{F}$.Bi'.

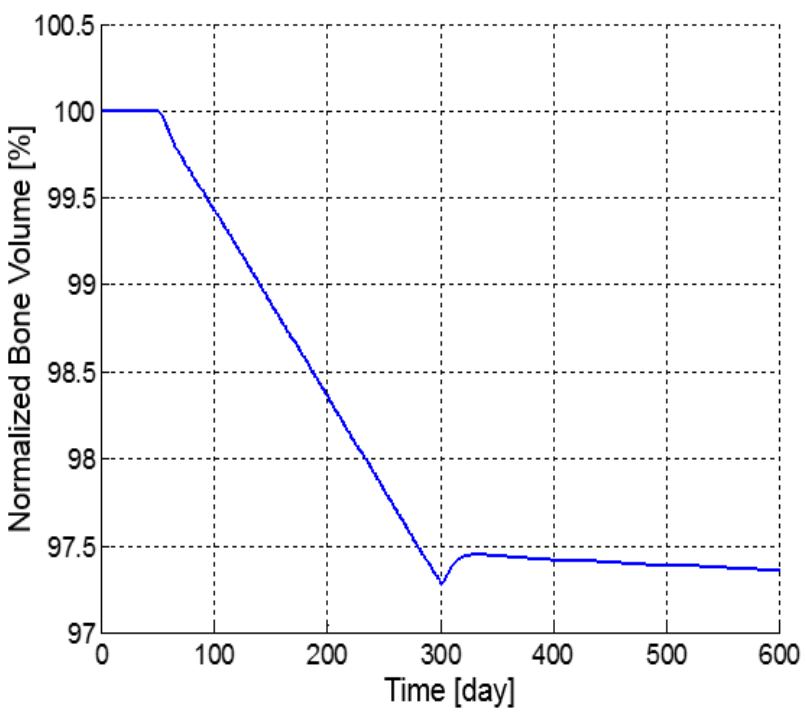

Fig. 7. The variation of normalized cell concentrations and bone volume with respect to their initial value during different periods: the normal (healthy) period from day 1 to day 50, the invasion of MM cells from day 51 to day 300 and the intervention of the bisphosphonates therapy from day 301 to day 600 ( $\mathrm{F} . \mathrm{Bi}=0.7)$.

\section{Conclusion}

In this paper, a computational model of MM-induced bone disease developed in our previous work [14] is extended to simulate the underlying mechanism of the bisphosphonates treatment. The extended computational model is able to simulate how the invasion of MM cells disturbs the stable state of the bone microenvironment and causes the fluctuation of cell concentrations and bone volume, and how the bisphosphonates treatment helps cell concentrations and bone volume return to their normal (healthy) values. The model results compare with published experimental data well and also demon- 
strate that the bisphosphonates treatment is effective in the management of MM-induced bone disease, since it can not only suppress osteoclast activity and the resultant bone resorption, but also has antitumour effects which then helps to get rid of the controversy whether the bisphosphonates treatment has anti-tumour effects.

\section{Acknowledgement}

This work was partly supported by the National Natural Science Foundation of China through grant 81301294, Post-doctorate Innovation Foundation of SHANDONG Province through grant 201303089 and the Independent Innovation Foundation of SHANDONG University through grant 2013HW009.

\section{References}

[1] J.A. Fowler, C.M. Edwards and P.I. Croucher, Tumor-host cell interactions in the bone disease of myeloma, Bone 48 (2011), 121-128.

[2] U. Heider, L.C. Hofbauer, I. Zavrski, M. Kaiser, C. Jakob and O. Sezer, Novel aspects of osteoclast activation and osteoblast inhibition in myeloma bone disease, Biochem. Biophys. Res. Commun. 338 (2005), 687-693.

[3] K.C. Nau and W.D. Lewis, Multiple myeloma: diagnosis and treatment, Am. Fam. Physician 78 (2008), 853-859.

[4] G.D. Roodman, Pathogenesis of myeloma bone disease, Blood Cells, Molecules \& Diseases 32 (2004), $290-292$.

[5] T. Matsumoto and M. Abe, TGF-beta-related mechanisms of bone destruction in multiple myeloma, Bone 48 (2011), $129-134$.

[6] G. Shay and M. Rogers, Bisphosphonates and cancer: current controversies, Osteoporosis Review 19 (2011), 9-12.

[7] R. T. Chlebowski, Z. Chen, J.A. Cauley et al., Oral bisphosphonate use and breast cancer incidence in postmen-opausal women, J Clin Oncol. 28 (2010), 3582-3590.

[8] R. Coleman, The use of bisphosphonates in cancer treatment, Bisphosphonates and Osteonecrosis of the Jaw 1218 (2011), 3-14.

[9] P. Musto, M.T. Petrucci, S. Bringhen et al., A multicenter, randomized clinical trial comparing zoledronic acid versus observation in patients with asymptomatic myeloma, Cancer 113 (2008), 1588-1595.

[10] S.V. Komarova, R.J. Smith, S.J. Dixon, S.M. Sims and L.M. Wahl, Mathematical model predicts a critical role for osteoclast autocrine regulation in the control of bone remodeling, Bone 33 (2003), 206-215.

[11] V. Lamaire, F.L. Tobin, L.D. Greller, C.R. Cho and L.J. Suva, Modelling the interactions between osteoblast and osteoclasts activities in the bone remodeling, J. Theoret. Biol. 229 (2004), 293-309.

[12] C. Rattanakul, Y. Lenbury, N. Krishnamara and D.J. Wollkind, Modeling of bone formation and resorption mediated by parathyroid hormone: response to estrogen/PTH therapy, Biosystems 70 (2004), 55-72.

[13] B. Ji, Mathematical modelling of bone remodelling at the cellular level and the interaction between myeloma cells and the bone microenvironment, PhD Dissertation, Univerisity of Hull, 2012.

[14] B. Ji, P.G. Genever, R.J. Patton and M.J. Fagan, Mathematical modelling of the pathogenesis of multiple myelomainduced bone disease, International Journal for Numerical Methods in Biomedical Engineering, 2014. (in press) doi: 10.1002/cnm.2645.

[15] B. Ji, D. Putra, R.J. Patton, P.G. Genever and M.J. Fagan, A novel mathematical model of bone remodelling cycles for trabecular bone at the cellular level, Biomechanics and Modelling in Mechanobiology 11 (2012), 973-982.

[16] V. M. Lauta, Interleukin-6 and the network of several cytokines in multiple myeloma: an overview of clinical and experimental data, Cytokine 16 (2001), 79-86.

[17] E. Terpos and M.A. Dimopoulos, Myeloma bone disease: pathophysiology and management, Annals of Oncology 16 (2005), 1223-1231.

[18] M. J. Rogers, S. Gordon, H. L. Benford, F. P. Coxon, S. P. Luckman, J. Monkkonen and J. C. Frith, Cellular and molecular mechanisms of action of bisphosphonates, Cancer 88 (2000), 2961-2978.

[19] G. Shay and M. Rogers, Bisphosphonates and cancer: current controversies, Osteoporosis Review 19 (2011), 9-12. 